\title{
Conducting a rapid review for quick turnaround knowledge synthesis
}

\author{
Mashrur Rahman Kazi ${ }^{1}$, Nashit Chowdhury ${ }^{1}$, Mohammad MZI Chowdhury ${ }^{1}$ and Tanvir C Turin ${ }^{1,2 *}$ \\ ${ }^{1}$ Department of Family Medicine, University of Calgary, Calgary, Alberta, Canada \\ ${ }^{2}$ Department of Community Health Sciences, University of Calgary, Calgary, Alberta, Canada
}

\begin{abstract}
The increase of information in recent years have led to different types of information synthesis such as systematic or literature reviews. In many circumstances, this information synthesis needs to be expedited in order to meet the rushed needs of practitioners, researchers and decision makers. In these scenarios, a rapid review can be deemed beneficial for the immediate need for acquiring information. A rapid review can be defined as a type of knowledge acquisition and synthesis (much like systematic reviews) where certain elements of the systematic review are simplified and/or omitted, such that information can be gathered in a time sensitive manner. A step-by-step guideline is provided in this paper as an introduction to those who are interested in conducting rapid reviews in order to meet the time sensitive needs of information. To help better understand the intricacies to conducting a rapid review, each step of the process is clearly explained and elaborated. Each step is also followed by examples from published literature. The intended audience includes students and investigators new to rapid reviews.
\end{abstract}

\section{Introduction}

In recent years, the increase of information has led to the creation of methods of information synthesis, such that massive volumes of information can be acquired and organized in a pragmatic and useful manner [1]. Literature reviews or systematic reviews serve this purpose by synthesizing existing information on a clearly formulated question through an explicit method to identify, select, extract and analyze relevant research works [2]. This type of synthesis contributes to acquire a deeper and comprehensive understanding of the issue of interest and is useful to provide unbiased evidence for practice and policy making. Generally, any well-done review would be undertaken through the following phases as shown in Figure 1.

In many instances the need arises where information is required to be synthesized in an expedited way, in order to meet the rushed needs of practitioners and policy makers. Sometimes the researchers themselves also need to explore information in a tight turnaround timeline in order to move their program of research forward. For example, the 2020 COVID-19 outbreak has created a scenario where a rapid synthesis of information was shown to be crucial $[3,4]$. During the circumstances of a global pandemic, it is necessary to accrue as much relevant health and disease information as possible in a short period of time, such that important health and safety guidelines can be implemented by policy

Table 1. Differences between Rapid Review and Systematic Review

\begin{tabular}{|l|l|}
\hline Rapid Review & Systematic Review \\
\hline $\begin{array}{l}\text { Time frame and duration of study is often } \\
\text { restricted }\end{array}$ & No hard restrictions on timeframe \\
\hline $\begin{array}{l}\text { May exclude hand-searching, limiting the } \\
\text { number of resources included in synthesis }\end{array}$ & $\begin{array}{l}\text { Exhaustive search is performed to include } \\
\text { relevant resources }\end{array}$ \\
\hline Broad capture of information & Focused capture of information \\
\hline $\begin{array}{l}\text { Identify and examine methodology of } \\
\text { research being studied }\end{array}$ & $\begin{array}{l}\text { Investigate and examine various results } \\
\text { and conflicts }\end{array}$ \\
\hline Optional bias assessment & Mandatory bias assessment \\
\hline Searches are limited by number of databases & Search is not limited \\
\hline
\end{tabular}

and decision makers, for the general public to follow in a rapid manner. When faced with immediate crises related to economic, business or health, a rapid synthesis of information can be deemed necessary for urgent decision making and handling of the situation in an appropriate manner. Also, there are certain situations where full exhaustive research is not required, but a rapid synthesis of information serves the purpose of acquiring information to support the cause. For example, rapid reviews can be beneficial for developing a research proposal or institutional decision making and guideline development.

Often, these types of needs for information can be served or fulfilled through conducting a rapid review. A rapid review can be defined as a type of knowledge acquisition and synthesis (much like systematic reviews). However, certain elements of the systematic review are simplified and/or omitted, such that information can be accrued in a time sensitive manner [5]. Rapid reviews have emerged as a streamlined approach to synthesize evidence in a timely manner, typically for the purpose of informing emergent decisions faced by decision makers in the healthcare setting [6]. Table 1 summarizes the differences between rapid reviews and systematic reviews.

\section{Steps of Conducting A Rapid Review}

In this manuscript, we will lay out in greater details how to conduct a rapid review. These steps (Figure 2) are described below with an example of a recently published rapid review from our research group. This rapid review was conducted to quickly synthesize evidence on spread and prevention of misinformation during large-scale disease

${ }^{*}$ Correspondence to: Tanvir C Turin MBBS Ms PhD, Department of Family Medicine, Room G012F, Health Sciences Center, 3330 Hospital Drive Northwest, Calgary, Alberta T2N 4N1, Canada, Tel: (403) 210-7199; Fax: (403) 210-9180; E-mail: turin.chowdhury@ucalgary.ca / dr.turin@gmail.com

Received: May 06, 2021; Accepted: June 11, 2021; Published: June 14, 2021 


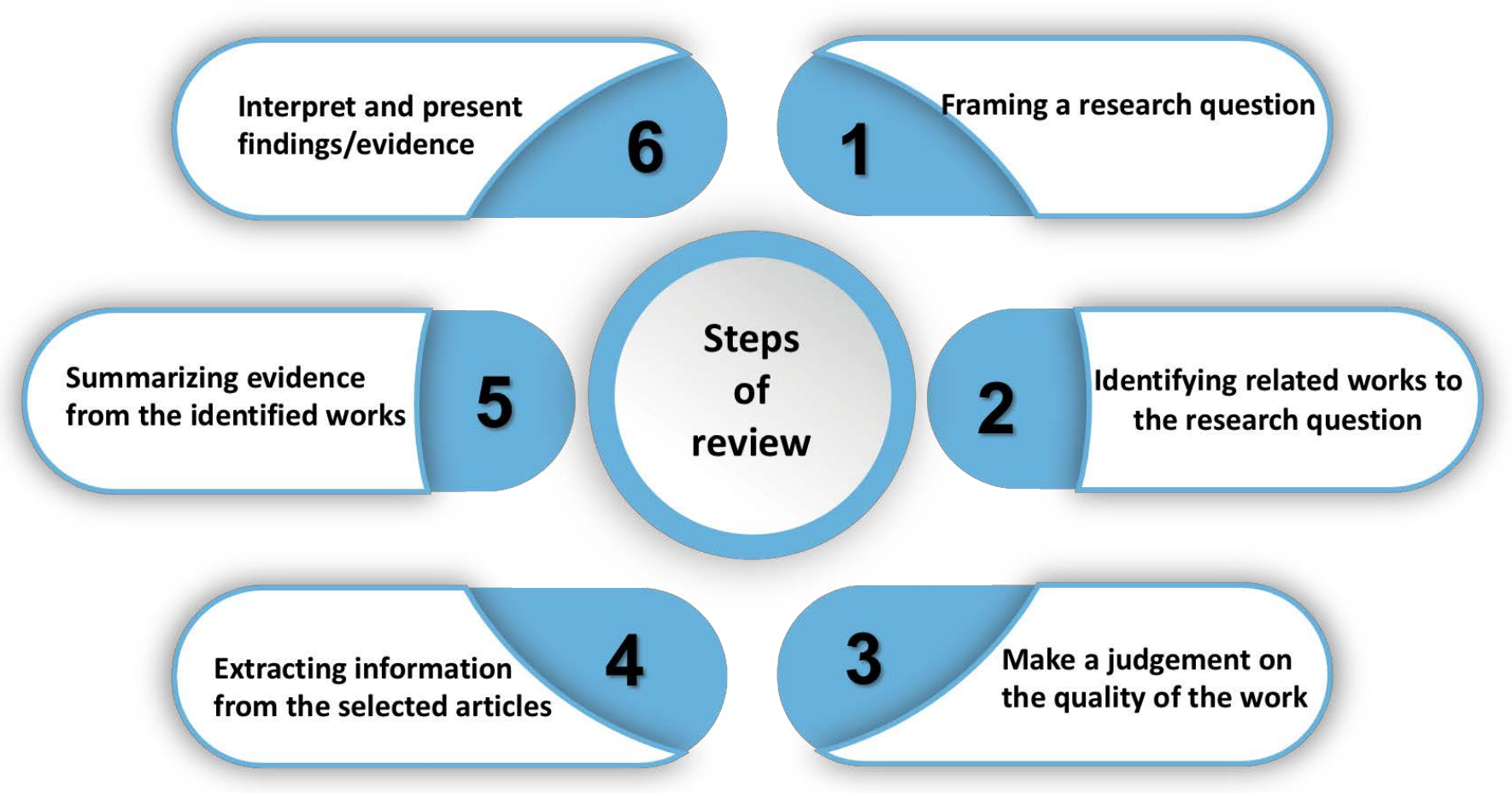

General Steps of Conducting Reviews

Figure 1. General steps of conducting a review

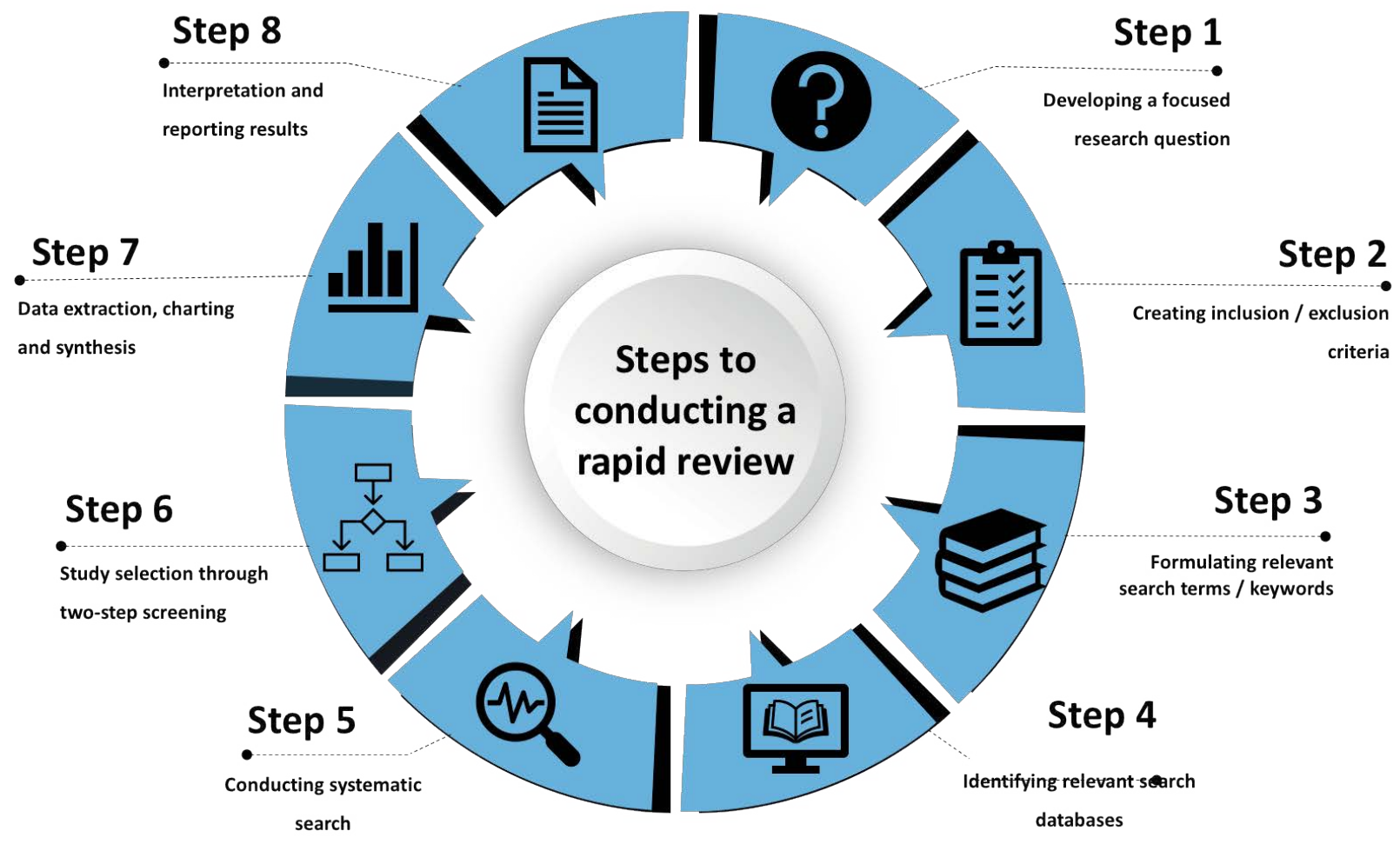

Steps of Conducting a Rapid Review

Figure 2. Steps to conducting a rapid review 


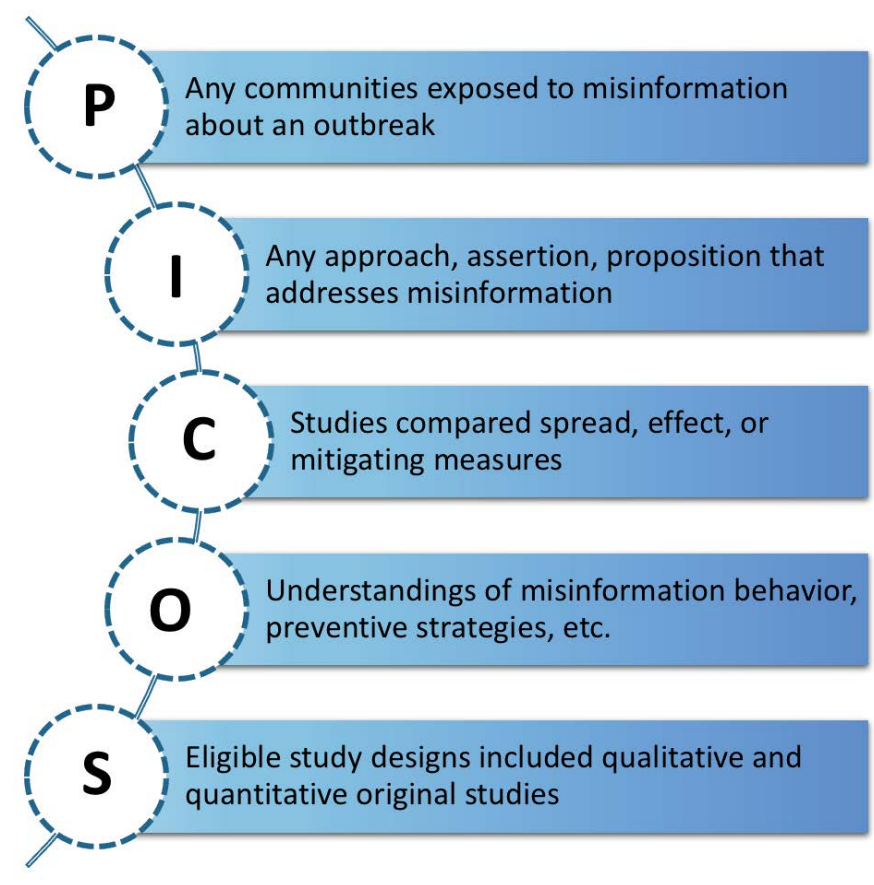

Figure 3. PICOS diagram from the example paper on misinformation infodemic [7]

outbreaks such as Ebola, COVID-19 and others [7]. We have obtained appropriate permission for reproducing these materials for this academic purpose.

\section{Step 1: Narrowing down on a research question}

The introductory step to executing a rapid review is to develop a research question based on the purpose, objectives and specific inquiries of the review. The development of the research question is a fundamental step in guiding the direction of the rapid review and dictating the methodology to follow. Following steps such as the development of the inclusion/exclusion criteria and timeframe of synthesis are guided by the research question. The principle focus of the review mold the breadth and depth of the rapid review.

One framework that helps guide the breadth and depth of the review is the PICOS framework. PICOS is an acronym for population, intervention, comparison, outcome, and study design and is often used for quantitative synthesis [8]. By employing these five components, investigators can narrow down their research focus and establish parameters of their inquiry. Narrowing down on a research question is the essential step for all following steps of conducting a rapid review (Figure 3).

\section{Step 2: Creating exclusion/inclusion criteria}

The creation of an inclusion and exclusion criteria establishes the parameters of a rapid review. It allows the investigators to capture the most relevant research work while eliminating irrelevant information. As systematic searches often yield a lot of information (both relevant and irrelevant to the research scope), the inclusion/exclusion criteria established by the investigators determine the comprehensiveness and specificity of the rapid review. It does so by guiding the investigators on what to include and what to exclude for the rapid review. Each established criterion must be clearly and rationally justified by the investigators, such that there is no confusion on the scope of the knowledge synthesis. Table 2 consists of the exclusion/inclusion criteria employed in the example rapid review.

\section{Step 3: Formulating relevant keywords}

Once the inclusion/exclusion criteria are established, the next step to conducting a rapid review is to formulate relevant keywords. This stage is crucial as it impacts the systematic search and therefore, the information that is yielded from the systematic search.

The first step to formulating keywords for a rapid review is to perform an initial limited search on the topic. This informal yet useful initial search of the databases allow investigators to screen the title and abstracts of related articles surrounding the general issue of interest. While screening, the investigators can identify relevant search terms used by these articles. Therefore, this initial search helps focus the rapid review as the most relevant search terms to the research question are being scoped out. This search also benefits the investigators by informing them about relevant databases as well. Overall, this initial limited search allows for a redefining of the search strategy.

Moreover, search terms required for the rapid review can be generally branched into two separate categories: keywords and subject headings. MeSH (Medical Subject Heading) terms are terminology used by the National Library of Medicine database in order to catalogue and index health related information [9]. These specific terms are used to index existing literature and therefore can yield relevant articles when identified as a search term in a systematic search. Furthermore, keywords are terms, words or phrases that can be used to search a database. In the absence of previously established MeSH terms, keywords can become extremely useful to capture relevant information.

In order to conduct a comprehensive and relevant systematic search for a rapid review, it is important to dissect each component of the PICOS diagram created for the research question. By breaking each component down, investigators can formulate relevant keywords

Table 2. The inclusion and exclusion criteria from the example paper on misinformation infodemic [7]

\begin{tabular}{|l|l|}
\hline Inclusion criteria & Exclusion criteria \\
\hline 1.Published in academic journals. & \\
2. Regarding misinformation & \\
3. Articles with misinformation in the & \\
communities about an abrupt large-scale & \\
infectious disease outbreak across different & \\
physical and virtual platforms & \\
4.(P) Populations: Any online or offline & \\
communities that were exposed, spread or & \\
used to spread misinformation about disease & 1.Related to infectious disease \\
outbreaks, or suffered due to misinformation & outbreaks not specific to our \\
during outbreaks & selection, such as HIV, malaria, etc. \\
5.(I) Interventions: Any approach, proposition, & 2.Mentioned misinformation as a \\
or assertion that fuels, evaluates, or fights & collateral outcome, but the research \\
misinformation during outbreaks & question was not designed to explore \\
6.(C) Comparison: Studies compared, evaluated, & misinformation. \\
assessed, or planned spread, effect, or & 3. Study designs other than original \\
mitigating measures for misinformation during & ones, such as reviews, organizational \\
an outbreak & reports, commentaries, letter to \\
7.(O) Outcomes: Outcomes included but & editors, and case studies \\
not limited to improved understanding of & 4. Studies not published in English \\
misinformation behaviour, the prevalence of & \\
misinformation, preventive strategies to correct & \\
misinformation & \\
8.(S) Study design: Eligible study designs & \\
included qualitative and quantitative original & \\
studies & \\
9. Time restriction was limited to the last 20 & \\
years (2001-2020) & \\
& \\
\hline
\end{tabular}


Table 3. The search terms developed for a rapid review from the example paper on misinformation infodemic [7]

\section{A.Misinformation-related search terms}

misinformation [keyword], disinformation [keyword], hoax [keyword], Deception [MeSH], rumo* [keyword], superstition [keyword, MeSH], misconception [keyword], misperception [keyword], fake news [keyword], false news [keyword], misleading information [keyword]

\section{B.Disease outbreak-related search terms}

"infectious disease" [keyword], Communicable disease [MeSH], virus [keyword], viruses [MeSH], outbreak [keyword], Disease Outbreaks [MeSH], Ebola [keyword], Ebola Vaccines [MeSH], Hemorrhagic Fever, Ebola [MeSH], Zika [keyword], Zika Virus [MeSH], Zika Virus Infection [MeSH], SARS [keyword], SARS virus [MeSH] Coronavirus Infections [MeSH], Betacoronavirus [MeSH], Coronavirus [keyword, MeSH], Severe Acute Respiratory Syndrome [MeSH], MERS [keyword], Middle East Respiratory Syndrome Coronavirus [MeSH], Swine flu [keyword], "Influenza A virus" [keyword], H1N1 Subtype [MeSH], COVID-19 [keyword]

Table 4. Search strategy for MEDLINE from the example paper on misinformation infodemic [7]

\begin{tabular}{|c|c|}
\hline \# & Searches \\
\hline 1 & misinformation.mp. \\
\hline 2 & disinformation.mp. \\
\hline 3 & hoax.mp. or exp Deception/ \\
\hline 4 & rumo*.mp. \\
\hline 5 & superstition.mp. or exp Superstitions/ \\
\hline 6 & misconception.mp. \\
\hline 7 & misperception.mp. \\
\hline 8 & fake news.mp. \\
\hline 9 & false news.mp. \\
\hline 10 & misleading information.mp. \\
\hline 11 & 1 or 2 or 3 or 4 or 5 or 6 or 7 or 8 or 9 or 10 \\
\hline 12 & infectious disease.mp. or exp Communicable Diseases/ \\
\hline 13 & virus.mp. or exp Viruses/ \\
\hline 14 & outbreak.mp. or exp Disease Outbreaks/ \\
\hline 15 & exp Hemorrhagic Fever, Ebola/ or exp Ebola Vaccines/ or Ebola.mp. \\
\hline 16 & exp Zika Virus Infection/ or exp Zika Virus/ or Zika.mp. \\
\hline 17 & $\begin{array}{l}\text { exp Betacoronavirus/ or exp Coronavirus Infections/ or exp SARS Virus/ or } \\
\text { SARS.mp. or exp Coronavirus/ or exp Severe Acute Respiratory Syndrome/ }\end{array}$ \\
\hline 18 & MERS.mp. or exp Middle East Respiratory Syndrome Coronavirus/ \\
\hline 19 & exp Influenza A Virus, H1N1 Subtype/ or Swine Flu.mp. \\
\hline 20 & COVID-19.mp. \\
\hline 21 & 12 or 13 or 14 or 15 or 16 or 17 or 18 or 19 or 20 \\
\hline 22 & 11 and 21 \\
\hline
\end{tabular}

Table 5. Search strategy for Google Scholar from the example paper on misinformation infodemic [7]

\section{Google Scholar search strategy}

\section{Search 1:}

\section{misinformation}

AND

("infectious disease" OR "communicable disease" OR virus OR outbreak OR Ebola OR Zika OR SARS OR Coronavirus OR "Severe Acute Respiratory Syndrome" OR MERS OR "Middle East Respiratory Syndrome" OR "Swine flu" OR "H1N1 virus" OR COVID-19)

\section{Search 2:}

disinformation

AND

("infectious disease" OR "communicable disease" OR virus OR outbreak OR Ebola OR Zika OR SARS OR Coronavirus OR "Severe Acute Respiratory Syndrome" OR MERS OR "Middle East Respiratory Syndrome" OR "Swine flu" OR "H1N1 virus" OR COVID-19)

Search 3:

misperception

AND

("infectious disease" OR "communicable disease" OR virus OR outbreak OR Ebola OR Zika OR SARS OR Coronavirus OR "Severe Acute Respiratory Syndrome" OR MERS OR "Middle East Respiratory Syndrome" OR "Swine flu" OR "H1N1 virus" OR COVID-19)

Search 4:

("fake news")

AND

("infectious disease" OR "communicable disease" OR virus OR outbreak OR Ebola OR Zika OR SARS OR Coronavirus OR "Severe Acute Respiratory Syndrome" OR MERS OR "Middle East Respiratory Syndrome" OR "Swine flu" OR "H1N1 virus" OR COVID-19) within each component and then later combine those using Boolean operators to yield a comprehensive systematic search [Table 3].

\section{Step 4: Identifying relevant search databases}

In contrast to a systematic review, rapid reviews use a minimum number of databases. We suggest that at least one appropriate academic database and one grey literature database should be chosen to conduct the search. As there are several databases available to search, the choice of the databases should be based on the context of the topic of interest. For example, if a rapid review is planned to be conducted on a very clinical topic, using MEDLINE or Embase should suffice. On the contrary, if it is a social science related topic, then the search should be conducted using CINAHL or ProQuest. Regarding grey literature databases, choosing Google Scholar is deemed enough for a rapid review.

\section{Step 5: Conducting systematic search}

After compiling the relevant search terms and the relevant search databases (grey and academic), a search strategy is created in order to yield the highest number of relevant information from the systematic search. We suggest a search strategy that is reflective of the research question and is exhaustive in nature, such that relevant information can be captured and synthesized for the rapid review.

Search strategies differ based on the academic database that has been employed for the systematic search. Generally, the search terms formulated for each component of the research question (as guided by PICOS in step 1) are combined in two steps. First, the search terms formulated within each research component are combined with the Boolean operator "OR" in order to capture the full scope of each individual research component. Once stringed by the Boolean operator "OR", the search results are then combined using the Boolean operator "AND" to conduct a systematic search that emphasizes each component of the research question and yields relevant results for inclusion in the rapid review. Table 4 and Table 5 provide examples of conducting a search through utilizing search terms in MEDLINE, which is one of the most popular databases in medical research and in Google Scholar, which is the most common search engine for grey literature, respectively.

The search strategy for grey literature mostly focuses on keyword searching. For a specific issue of inquiry, investigators combine relevant universal keywords in the search bar of the database by using Boolean operators "AND" and "OR". Once these search terms are combined, they can be used to search grey literature databases such as Google Scholar. In addition, only the first 100 results or the first 10 pages are taken into consideration for the rapid review because of how the search algorithm generally show the most relevant papers or websites at the top of the list. In the example (Table 5) a series of cumulative searches were used due to the limitation of characters in the search box. The results of the first 10 pages (100 results) for each search were selected.

\section{Step 6: Study selection through Two-step screening}

After being yielded from the systematic search, relevant articles are identified through a two-step screening process that allows the 


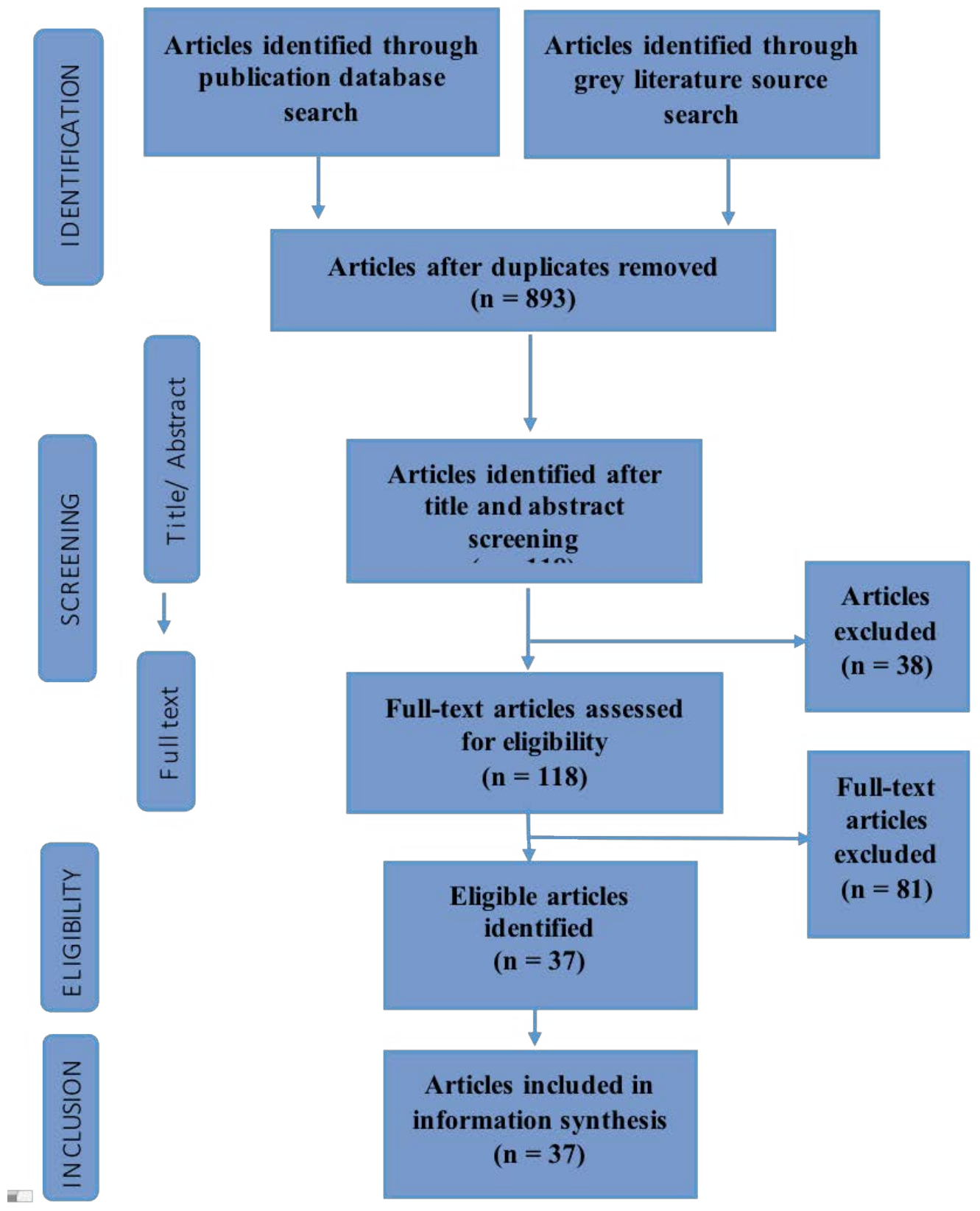

Figure 4. Literature search and selection schematic from the example paper on misinformation infodemic [7]

investigators to identify the most relevant information according to the established research parameters. The first step of study-selection includes screening the title and abstract of the articles that were chosen through the systematic search. In contrast to the conventional systematic review, rapid review's screening is conducted by a single reviewer. If there is any doubt regarding the relevancy of the article to the research question, the reviewer must include the study for a full-text screen. If the title and abstract of the articles screened in the previous step can meet the inclusion criterion set by the investigators, then the article can proceed to the second stage of the study selection which is the fulltext screening. As the name suggests, full-text screening consists of the investigators screening and analyzing the entirety of the research work at question. Though not mandatory, investigators may manually go through the reference lists or bibliography of the final selected papers, in order to identify if any other relevant papers have been missed. This technique is defined as Snowballing, Pearl Growing or Citation Mining [10]. Software such as Covidence or MS Excel is often used to aid with the process of this screening [Figure 4].

\section{Step 7: Data extraction, charting and synthesis}

Once the research work to include in the rapid review is selected, the key points of information from these works need to be extracted and charted accordingly. Information collected can be presented in a multitude of ways in a rapid review. Result charting and presentation vary from review to review and is reflective of the research question. Generally, information collected can be presented in tables, charts, graphs and diagrams. They are often presented in a logical manner such that the reader can understand the key information. At large, the first table normally presents the following 


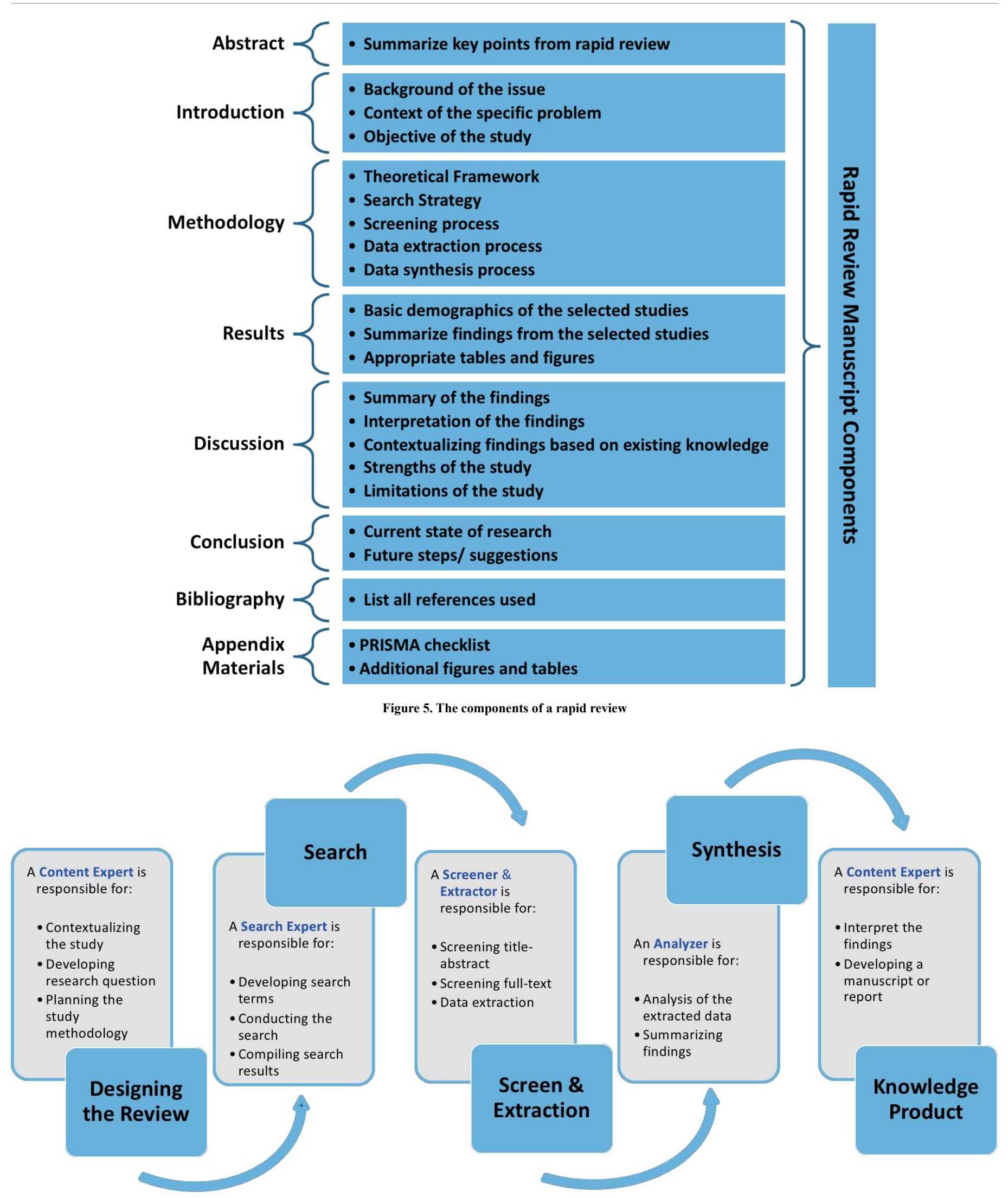

Roles and Responsibilities of Rapid Review Team

Figure 6. The roles and responsibilities of a rapid review team 
fundamental information regarding the research works included in the rapid review; year of publication, the demographic information, author, country of research, type of research, etc. The charts and/or tables to follow are completely dependent on the investigators' preferences on how they would like to express the findings of the rapid review.

\section{Step 8: Interpretation and reporting results}

Information extracted from the research works need to be interpreted, summarized and presented in a logical manner such that it surrounds the main research question while clearly reporting the findings from the different information resources. In the summarization of information included in the rapid review, investigators need to clearly describe the purposes and findings of the research works included. Once the information has been extracted, charted and synthesized, it is the investigator's responsibility to thematically categorize the information such that it is presented in a manner that aligns with the research aim of the rapid review. This thematic categorization is reflective of the findings from the rapid review and therefore can vary from review to review.

\section{Creating the knowledge product as a manuscript}

The final step of the process of conducting a rapid review is to draft a manuscript for submission to a journal.

\section{Preferred reporting checklist for rapid reviews}

Scoping reviews and systematic reviews often employ a checklist known as Preferred Reporting Items for Systematic reviews and MetaAnalyses (PRISMA). This checklist has been developed to make sure that a minimum set of items are met by investigators in order to improve the reporting of systematic reviews and meta-analyses [11]. However, a PRISMA checklist for rapid reviews has not been established yet and is currently under development [12]. The components of a rapid review manuscript is shown in Figure 5.

\section{Creating A Team}

Conducting a successful rapid review requires the development and assembly of a review team based on varied skill sets. As there are different components that need to be completed for the rapid review, individuals with skill sets corresponding to these components are required to be present in the team. A screener with expertise in the methodological and content direction is required in a rapid review team. The team can be expanded with an experienced librarian who can take on the comprehensive search process. Together, the members of the team are responsible for determining the breadth and depth of the rapid review. The team must also address potential limitations of the rapid review. The following roles and responsibilities are needed to be incorporated for a successful rapid review (Figure 5 and 6).

\section{Conclusion}

Rapid reviews act as a proficient and useful tool under circumstances where information needs to be summarized in an expedited manner. Given the accelerated nature and process of conducting a rapid review, decision makers, practitioners and researchers can employ this rapid form of information synthesis in order to meet their rushed needs. Therefore, rapid reviews can be extremely beneficial in immediate information acquisition situations such as global pandemics, economic crises and research done under a tight timeline.

\section{Conflict of Interest}

There is no conflict of interest.

\section{References}

1. Ahmed S, Vaska M, Turin T (2016) Comprehensive systematic search process of health literature: hunting pearls out of the sea. J Natl Hear Found Bangladesh 5: 12-16.

2. Sofaer N, Strech D (2011) The Need for Systematic Reviews of Reasons. Bioethics 26 315-328. [Crossref]

3. Khangura S, Konnyu K, Cushman R (2012) Evidence summaries: The evolution of a rapid review approach. Syst Rev 1: 10.

4. Khangura S, Polisena J, Clifford TJ, Farrah K, Kamel C, et al. (2014) Rapid review: An emerging approach to evidence synthesis in health technology assessment. Int $J$ Technol Assess Health Care 30: 20-27. [Crossref]

5. Tricco AC, Antony J, Zarin W, Strifler L, Ghassemi M, et al. (2015) A scoping review of rapid review methods. BMC Med 13: 224. [Crossref]

6. Davies C, Fattori F, O'Donnell D, Donnelly S, Shé EN, et al. What are the mechanisms that support healthcare professionals to adopt assisted decision-making practice? A rapid realist review. BMC Health Serv Res 19: 960. [Crossref]

7. Chowdhury N, Khalid A, Turin TC (2021) Understanding misinformation infodemic during public health emergencies due to large-scale disease outbreaks: a rapid review. $J$ Public Health (Berl).

8. Methley AM, Campbell S, Chew-Graham C, McNally R, Cheraghi-Sohi S (2014) PICO, PICOS and SPIDER: A comparison study of specificity and sensitivity in three search tools for qualitative systematic reviews. BMC Health Serv Res 14: 579. [Crossref]

9. https://www.nlm.nih.gov/bsd/disted/meshtutorial/introduction/index.html

10. https://ibguides.mssm.edu/c.php?g=168555\&p=1107625

11. Moher D, Liberati A, Tetzlaff J, Altman DG, Group P, et al. (2009) Preferred reporting items for systematic reviews and meta-analyses: The PRISMA statement. PLoS Med 6: e1000097. [Crossref]

12. Stevens A, Garritty C, Hersi M, Moher D (2018) Developing PRISMA-RR, a reporting guideline for rapid reviews of primary studies (Protocol). 2018: 1-12.

Copyright: (C2021 Turin TC. This is an open-access article distributed under the terms of the Creative Commons Attribution License, which permits unrestricted use, distribution, and reproduction in any medium, provided the original author and source are credited. 